\title{
The comparative anatomy of the folds, fossae, and adhesions around the duodenojejunal flexure in mammals
}

\author{
M. Ishida, N. Sakata, I. Ise, T. Ono, M. Shimura, K. Ishii, M. Murakami, T. Takadate, \\ T. Aoki, K. Kudo, S. Ohnuma, K. Fukase, H. Ohtsuka, M. Mizuma, H. Hayashi, \\ K. Nakagawa, T. Morikawa, F. Motoi, T. Naitoh, M. Unno
}

Department of Surgery, Tohoku University Graduate School of Medicine, Aobaku, Japan

[Received: 30 April 2017; Accepted: 20 September 2017]

Background: Anatomical knowledge of the duodenojejunal flexure is necessary for abdominal surgeries, and also important for physiologic studies about the duodenum. But little is known about the anatomy of this region in mammals. Here, we examined comparative anatomy to understand the anatomical formation of the duodenojejunal flexure in mammals.

Materials and methods: The areas around the duonenojejunal flexure were observed in mouse, rat, dog, pig, and human, and the anatomical structures around the duodenojejunal junction in the animals were compared with those in human. Results: The superior and inferior duodenal folds, and the superior and inferior duodenal fossae were identified in all examined humans. In pig, the structures were not clearly identified because the duodenum strongly adhered to the retroperitoneum and to the mesocolon. In mouse, rat, and dog, only the plica duodenocolica, which is regarded as the animal counterpart of the superior duodenal fold in human, was identified, and other folds or fossae were not observed, probably because the duodenum was not fixed to the parietal peritoneum in those animals. Transection of the plica duodenocolica could return the normally rotated intestine back to the state of non-rotation in rat.

Conclusions: This study showed the anatomical similarities and dissimilarities of the duodenojejunal flexure among the mammals. Anatomical knowledge of the area is useful for duodenal and pancreatic surgeries, and for animal studies about the duodenum. (Folia Morphol 2018; 77, 2: 286-292)

Key words: duodenojejunal flexure, flexura duodenojejunalis, duodenal fossa, plica duodenocolica, suspensory muscle of the duodenum, and superior duodenal fold

\section{INTRODUCTION}

The duodenojejunal flexure (flexura duodenojejunalis) is defined as the border between the duodenum and the jejunum. The anatomy around the dudenojejunal flexure is important for duodenal and pancreatic surgeries, especially for pancreatoduodenectomy, one of the most invasive and difficult procedures in digestive surgery, but the anatomy is complex and difficult to understand in human. In contrast to human, the anatomy of the duodenojejunal flexure is rather sim-

Address for correspondence: Dr. M. Ishida, Department of Surgery, Tohoku University Graduate School of Medicine, 1-1 Seiryomachi, Aobaku, Sendai 980-8574, Japan, tel: +81-22-717-7205, fax: +81-22-717-7209, e-mail: mishida@surg1.med.tohoku.ac.jp 
ple in animals, because the duodenum possesses its own mesentery, mesoduodenum. The duodenum in animals is not defined by the presence of the duodenal glands as in human, and the border between the duodenum and the jejunum is marked by the plica duodenocolica (PDC) in animals [11].

The structures around the duodenojejunal flexure of human generally consist of 2 folds and 2 fossae. The superior duodenal fold (SDF), also called the duodenojejunal fold or plica duodenalis superior, is a serous, membranous fold of the peritoneum, abutting on the superior duodenal fossa [10]. The inferior mesenteric vein runs on the left side of the SDF, and the right side of the SDF connects to an adhesion between the duodenojejunal flexure and the transverse mesocolon [3]. SDF is used as a reference point for the end of the duodenum and the beginning of the jejunum in abdominal surgery, and is sometimes confused with the suspensory muscle of the duodenum, i.e. the ligament of Treitz [16], which connects the third and fourth portion of the duodenum and the connective tissue between the coeliac and the superior mesenteric artery [9], and is the main cause of the superior mesenteric artery syndrome in human [1].

The inferior duodenal fold (IDF, also called the duodenomesocolic fold or plica duodenalis inferior) is also a serous, membranous fold of the peritoneum that connects the duodenum and the descending mesocolon, and abuts on the inferior duodenal fossa [10].

The superior and inferior duodenal fossae are constructed by the duodenum, the mesocolon, and the superior and inferior duodenal fold, the bottom of which is the parietal peritoneum. However, there are slight differences in their definition among researchers $[2,4,10,12]$. They are known as common sites for a paraduodenal hernia, one of the abdominal internal hernias [8].

In animals, one of the anatomical structures around the duodenojejunal flexure is the PDC. The fold is present in domestic animals, such as pig, cow, horse, and dog [7, 11], and it demarcates the border between the duodenum and the jejunum. However, the anatomical counterparts of the SDF, the IDF, and the superior and inferior duodenal fossae, are not clarified, and, to our knowledge, differences in the anatomy around the duodenojejunal flexure between human and other animals have not been analysed. Anatomical information about the duodenojejunal flexure in mammals is necessary for understanding the generalised pattern and developmental processes of the area, and would also facilitate surgical and physiological experiments in mammals.

Here, we provide anatomical information about the duodenojejunal flexure of four major experimental animals, mouse, rat, dog, and pig, in relation to that of human.

\section{MATERIALS AND METHODS}

\section{Ethics statement}

The present study was approved by the Research Ethics Committee of Tohoku University, School of Medicine (2016-1-064), and patient anonymity was preserved.

\section{Human study}

Ten surgical records of pancreatoduodenectomy performed at Tohoku University Hospital from 2013 to 2015 were reviewed to observe the anatomy of the duodenojejunal flexure. The appearance of the superior and inferior duodenal fossae, SDF, and IDF were noted.

\section{Experimental animals}

The animal subjects were 2 domestic male pigs at 3 months old (Kumagai-Shigeyasu Co., Ltd, Sendai, Japan), 2 Beagle dogs (1 male and 1 female) at 1.5 years old (Oriental Bio Service, Kyoto, Japan), 2 male Wistar rats at 2 months old (Kumagai-Shigeyasu Co., Ltd, Sendai, Japan), and 2 male BALB/c mice at 10 weeks old (CLEA Japan, Tokyo, Japan). All animals were euthanised before the abdominal exploration with intraperitoneal pentobarbital $(150 \mathrm{mg} / \mathrm{kg})$ for mice and rats, with intravenous thiopental sodium $(60 \mathrm{mg} / \mathrm{kg})$ for dogs, and with intravenous potassium chloride ( $120 \mathrm{mEq} / \mathrm{body}$ ) under general anaesthesia maintained with inhaled sevoflurane and oxygen for pigs.

\section{Comparative anatomy of the duodenojejunal flexure}

The abdomen was widely incised for peritoneal exploration. The duodenojejunal flexure in the experimental animals was identified by following the small intestine from the colon to the oral side. Because the duodenojejunal flexure is demarcated by the PDC $[7,11]$, the first fold or adhesion identified when progressing to the duodenum was regarded as the PDC. The duodenum was also observed from 

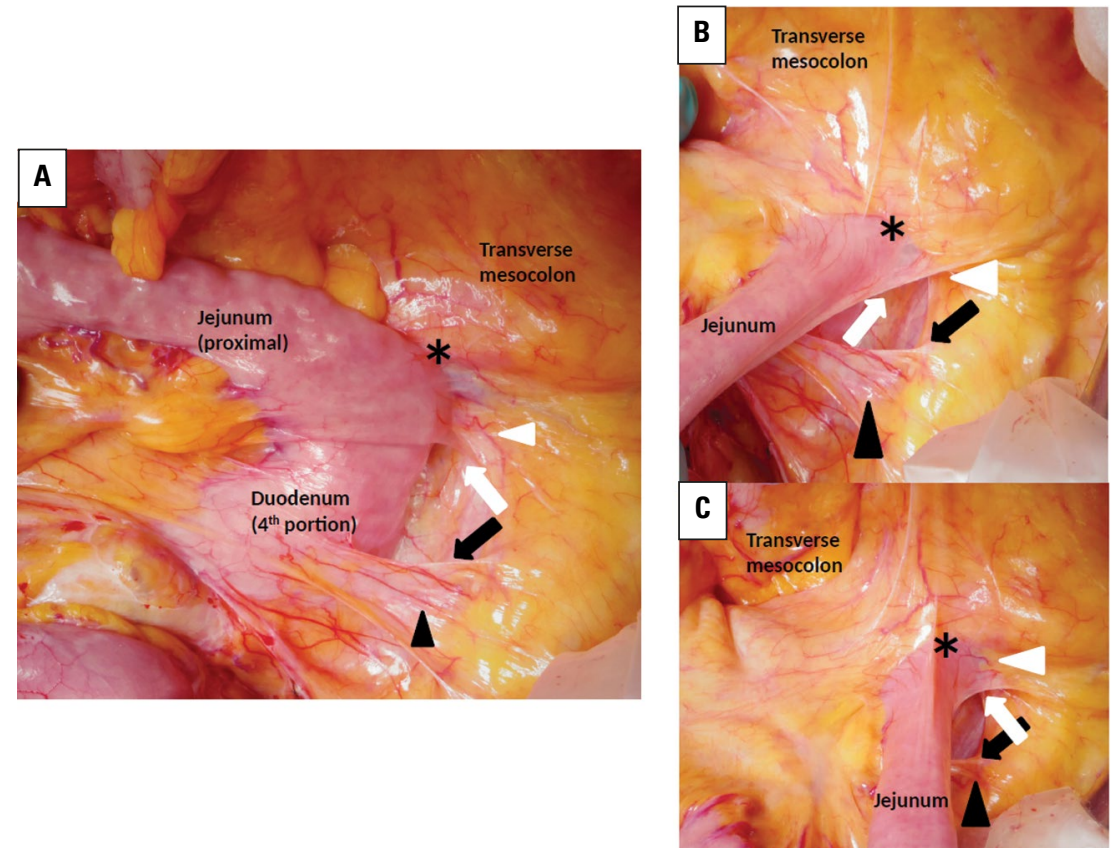

Figure 1. The duodenojejunal flexure of human; A. The jejunum is pulled to the right. The superior duodenal fold (white arrowhead) and the inferior duodenal fold (black arrowhead) can be clearly seen. The superior (white arrow) and inferior duodenal fossae (black arrow) are observed between the superior duodenal fold and the inferior duodenal fold. Only a part of the adhesion between the duodenojejunal flexure and the transverse mesocolon $\left(^{*}\right)$ can be seen in this view; B, C. The adhesion between the duodenojejunal flexure and the transverse mesocolon ${ }^{*}$ ) can be easily observed when the jejunum is pulled right-caudally (B) or caudally (C). The superior duodenal fold (white arrowhead) is connected to the adhesion, and their boundary is unclear. The inferior duodenal fold is indicated by a black arrowhead, and the superior duodenal fossa and the inferior duodenal fossa are indicated by white and black arrows, respectively.

the oral side by following the stomach. The beginning of the duodenum was identified by the pylorus, which is generally found in most vertebrates [13], and the duodenum was followed to the duodenojejunal flexure. Any peritoneal membranous folds around the duodenojejunal flexure were investigated, and when any duodenal fossa was detected, it was considered to be the superior or inferior duodenal fossa.

\section{Terminology}

The anatomical words used in this study were based on Terminologia Anatomica [5] for human, and Nomina Anatomica Veterinaria [17] for animals. The SDF, the IDF, the superior and inferior duodenal fossa, and the suspensory muscle of the duodenum are from Terminologia Anatomica [5], and the PDC is from Nomina Anatomica Veterinaria [17]. The explanations about "the adhesion between the duodenojejunal flexure and the mesocolon" and "the fixation of the duodenum to the retroperitoneum" are simple descriptions and were not from Terminologia Anatomica [5], or Nomina Anatomica Veterinaria [17].

\section{RESULTS}

\section{Human}

Figure 1 shows the representative anatomy of the duodenojejunal flexure of human. The SDF, IDF, and the superior and the inferior duodenal fossae were detected in all 10 cases. The SDF and IDF abutted on the superior and the inferior duodenal fossa, respectively. The inferior mesenteric vein could be seen through the serosa on the left side of the SDF.

The SDF connected to an adhesion between the duodenojejunal flexure and the transverse mesocolon (Fig. 1A). When the jejunum was pulled caudally, the fibrous adherence of the SDF and the connection to the adhesion could be observed (Fig. 1B, C). The SDF and the adhesion between the duodenojejunal flexure and the transverse mesocolon were transected in every case of pancreatoduodenectomy in order to detach the duodenum from the mesocolon and to approach the vessels of the duodenum and the pancreas. Lysis of the adhesion also exposed the suspensory muscle of the duodenum. 
The IDF was connected to the visceral peritoneum of the third portion of the duodenum, and it was also transected in every pancreatoduodenectomy.

\section{Pig}

Figure 2 shows the duodenojejunal flexure of pig. The arrangement of the intestines is different from that of human. The small intestine is located in the right abdomen and the large intestine in the left abdomen in pig, while the large intestine adheres to both sides of the abdominal wall in human. The duodenum of pig is fixed to the retroperitoneum like that of human, but the extent of the adhesion is different. While almost all the duodenum is fixed to the retroperitoneum in human, the oral side of the duodenum of pig is not fixed to the retroperitoneum, and the anal side of the duodenum strongly adheres to the retroperitoneum and to the mesocolon. A serous fold between the mesocolon and the duodenum was observed (Fig. 2), and it was identified as the PDC. The fold connected to the adhesion between the duodenojejunal flexure and the mesocolon. No other fold or fossa was observed. However, it is possible that anatomical structures, which are counterparts of the IDF, and the superior and inferior duodenal fossae of human, would be preserved in the strong adhesion, and we could not identify them macroscopically. The suspensory muscle of the duodenum was not identified in pig.

\section{Dog}

Figure 3 shows the duodenojejunal flexure of dog. Unlike in human and pig, the duodenum was not fixed to the parietal peritoneum. The PDC was easily detected by following the small intestine to the oral side, and the PDC was continuous with the adhesion between the duodenojejunal flexure and the mesocolon. The duodenum was not fixed to the retroperitoneum and no other folds or fossae were identified. The suspensory muscle of the duodenum was not macroscopically apparent in dog.

\section{Mouse and rat}

The two rodents showed nearly the same anatomy around the duodenojejunal flexure, and the placement of the bowels of the rodents was very similar to that of dog. Figure $4 \mathrm{~A}$ shows the duodenojejunal flexure of mouse, and Figure $4 \mathrm{~B}$ and $4 \mathrm{C}$ show that of rat. Just as in dog, the adhesion between the duodenojejunal flexure and the mesocolon was easily

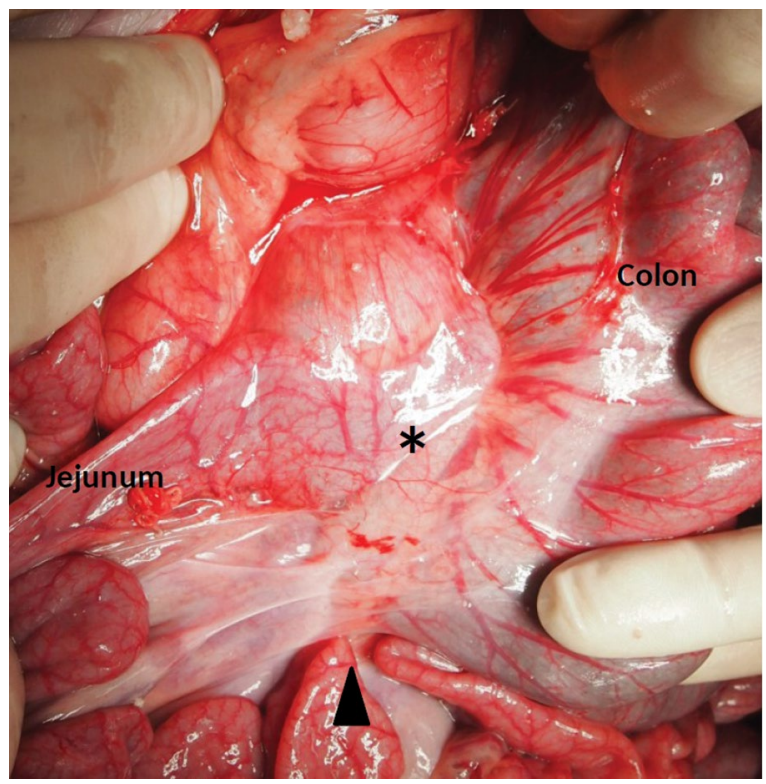

Figure 2. The duodenojejunal flexure of pig. The adhesion between the duodenojejunal flexure and the mesocolon was thick and strong $\left({ }^{*}\right)$. Black arrowhead indicates the plica duodenocolica. No recess-like structure could be identified.



Figure 3. The duodenojejunal flexure of dog. The adhesion between the duodenojejunal flexure and the mesocolon is indicated by an asterisk $\left({ }^{*}\right)$ and the plica duodenocolica can be seen on the left side of the adhesion (black arrowhead). Because the duodenum was not fixed to the parietal peritoneum, no other folds or fossae could be identified.

detected and, to the left of the adhesion, the PDC was identified. The duodenal fossae and other folds were not found, perhaps because the duodenum was not fixed to the parietal peritoneum. When the PDC and the adhesion were cut in rat, the naturally rotated intestine returned to a state of non-rotation (Fig. 5). No obvious muscular structure like the suspensory 


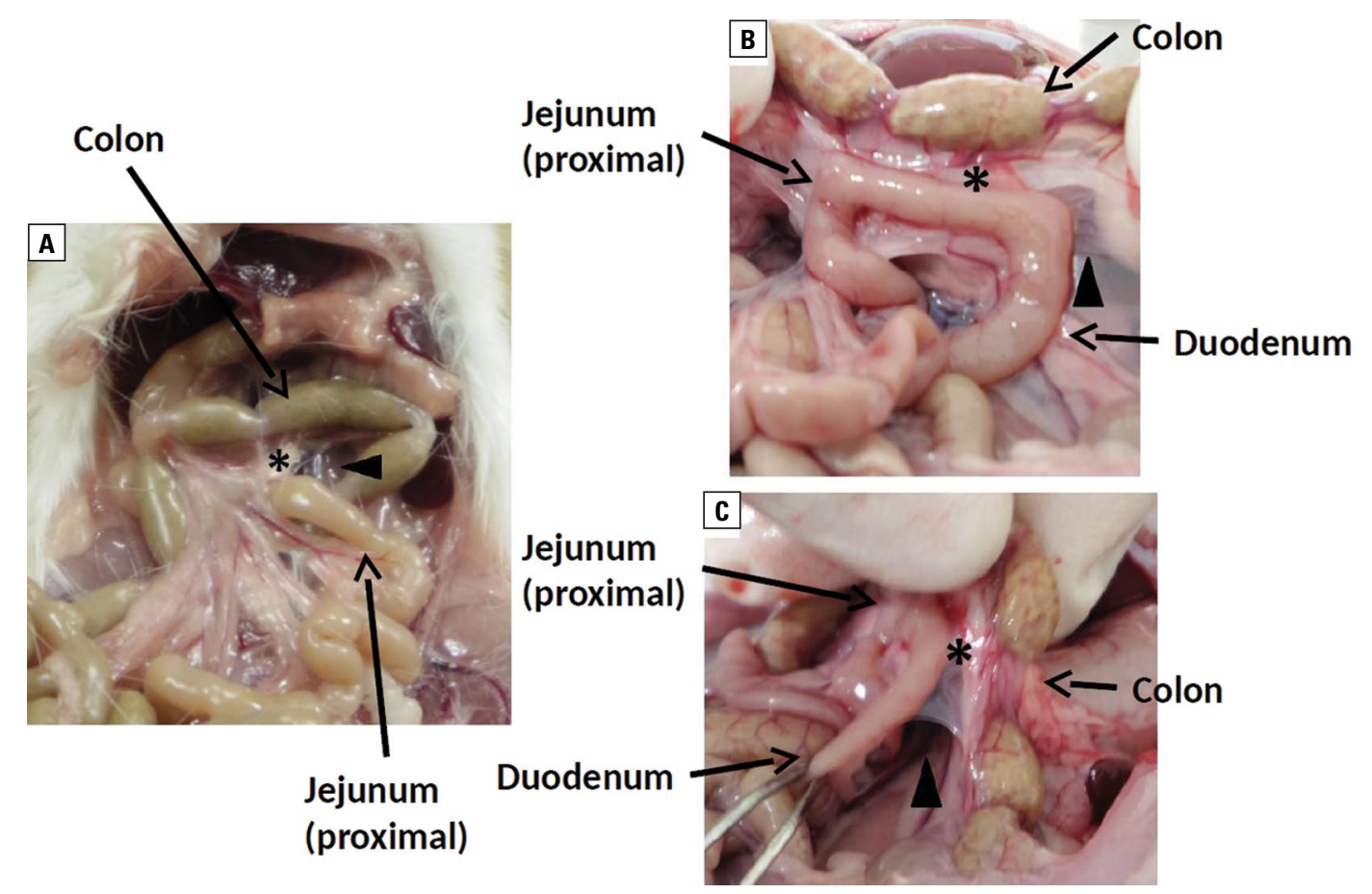

Figure 4. The duodenojejunal flexure of mouse and rat. Panel $\mathbf{A}$ shows the duodenojejunal flexure of mouse. The adhesion between the duodenojejunal flexure and the mesocolon is indicated by an asterisk $\left(^{*}\right)$. The plica duodenocolica was identified on the left edge of the adhesion (black arrowhead). Because the duodenum was not fixed to the parietal peritoneum, other folds or fossae could not be identified. The duodenojejunal flexure of rat is shown in panels $\mathbf{B}$ and $\mathbf{C}$. Panel $\mathbf{B}$ is a view from the front and panel $\mathbf{C}$ from the left. The anatomy around the duodenojejunal flexure is similar to that of mouse. The plica duodenocolica (black arrowhead) was identified on the left of the adhesion between the duodenojejunal flexure and the mesocolon $\left.{ }^{*}\right)$. Other folds or fossae were not identified, as in mouse.

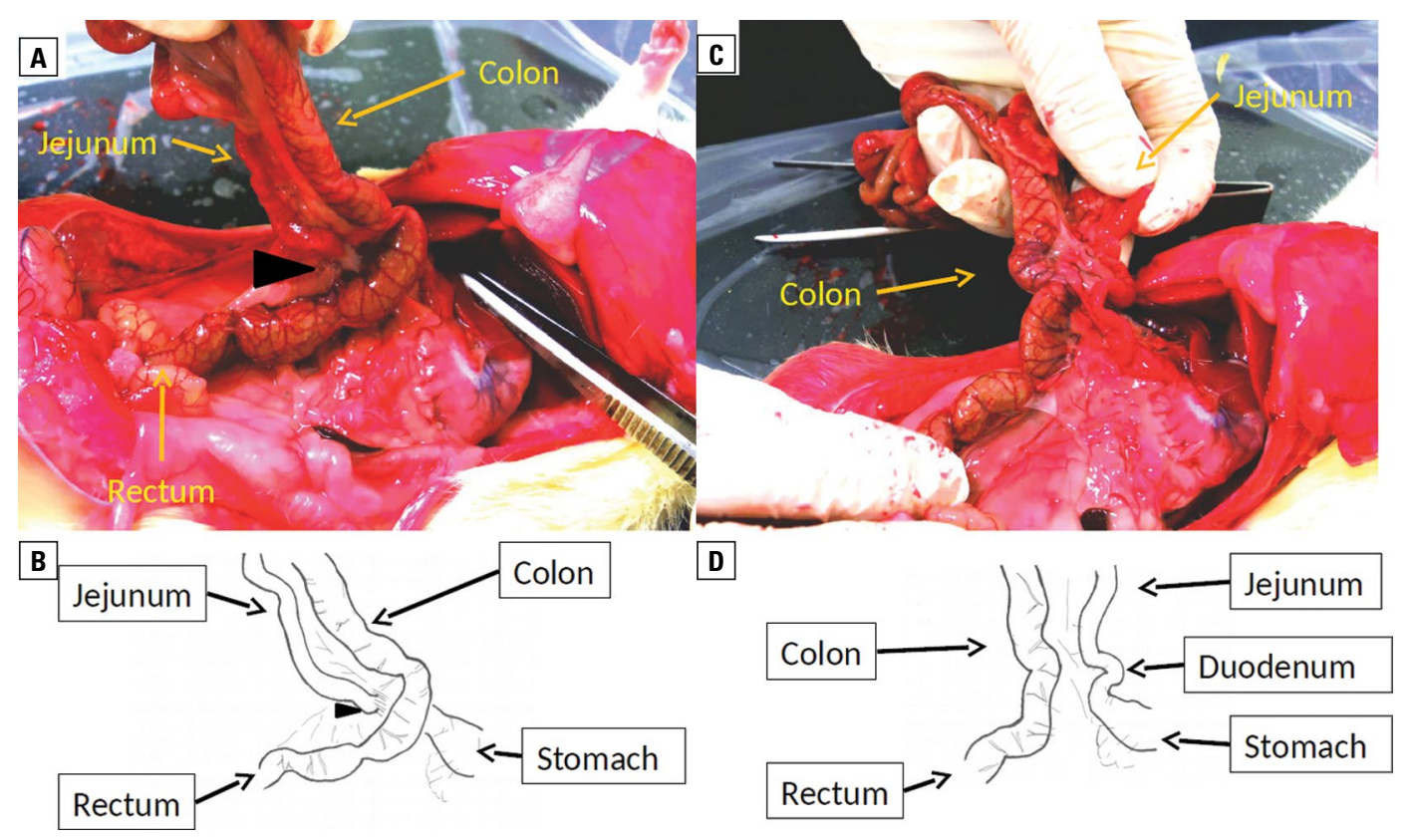

Figure 5. Repositioning of the intestine to a state of non-rotation. By cutting the plica duodenocolica (black arrowhead) and the adhesion between the duodenojejunal flexure and the mesocolon in rat, the physiologically rotated intestine was returned to a state of non-rotation; A, B. Before the transection. The ileum was elevated upward to show the physiologically rotated intestine. The duodenum is naturally rotated counterclockwise, and appears to have whirled the colon. Panel $\mathbf{A}$ is a photographic view and panel $\mathbf{B}$ is a schema for the view; C, D. After the transection. By cutting the adhesion, the duodenum could be turned clockwise, and the rotation returned to non-rotation. Panel $\mathbf{C}$ is a photographic view and panel $\mathbf{D}$ is the schema for the view. 
Table 1. The anatomical structures around the duodenojejunal flexure in mammals

\begin{tabular}{|c|c|c|c|c|c|}
\hline & Human & Pig & Dog & Mouse & Rat \\
\hline $\begin{array}{l}\text { Superior duodenal fold (SDF) } \\
\text { Plica duodenocalica (PDC) }\end{array}$ & $Y$ & Y & $Y$ & $Y$ & Y \\
\hline Inferior duodenal fold (IDF) & $Y$ & $\mathrm{~N}$ & N & $\mathrm{N}$ & $\mathrm{N}$ \\
\hline Superior duodenal fossa & $Y$ & $\mathrm{~N}$ & $\mathrm{~N}$ & $\mathrm{~N}$ & $\mathrm{~N}$ \\
\hline Inferior duodenal fossa & $Y$ & $\mathrm{~N}$ & N & $\mathrm{N}$ & $\mathrm{N}$ \\
\hline Suspensory muscle of duodenum & Y & N & $\mathrm{N}$ & $\mathrm{N}$ & $\mathrm{N}$ \\
\hline "Fixation" & $Y$ & Y & $\mathrm{N}$ & $\mathrm{N}$ & $\mathrm{N}$ \\
\hline "Adhesion" & $Y$ & $Y$ & $Y$ & $Y$ & Y \\
\hline
\end{tabular}

*SDF in human was regarded as the counterpart to PDC in animal. Y — yes; positive for the structure. N — no; negative for the structure; "Fixation" — fixation of the duodenum to the retroperitoneum; "Adhesion" — adhesion between the duodenojejunal flexure and the transverse mesocolon

muscle of the duodenum of human was observed macroscopically.

\section{Summary of the results}

We summarised the information about the folds and fossae around the duodenojejunal flexure from each animal in Table 1. The SDF in human was regarded as a counterpart of PDC in animals, because both folds demarcated the border between the duodenum and the jejunum, and because the adhesion between the duodenojejunal flexure and the mesocolon was identified next to the fold. These data revealed that there are significant differences in the anatomy of the duodenojejunal flexure between human and other animals.

\section{DISCUSSION}

Our study elucidated the preservation of the anatomical structures around the duodenojejunal flexure in the examined mammals (Table 1).

The adhesion between the duodenojejunal flexure and the mesocolon was common to all the animals, and it forms the SDF in human and the PDC in the other animals. That is the common characteristic of the duodenojejunal flexure, and may have embryologic and evolutionary importance. Based on the finding that lysis of the PCD and the adhesion between the duodenojejunal flexure and the mesocolon in rat changed the naturally rotated intestine into a state of non-rotation, they were assumed to have a role in the embryologic intestinal rotation. The intestine must be rotated in order to provide a sufficient absorptive area for the relatively short trunk [6], but the driving force of intestinal rotation is still unknown [14]. The PCD and the adhesion between the duodenojejunal flexure and the mesocolon is the essential structure to maintain the intestinal rotation, and possibly to commence the intestinal rotation during the prenatal period. Derotation of the intestine in human is not so simple in human as in rat, because, in addition to the lysis of the adhesion between the duodenojejunal flexure and the mesocolon, wide incision of the peritoneum is necessary to mobilise the duodenum [15].

The anatomical divergence of the duodenojejunal flexure among the animals is thought to derive from the differences of the duodenal fixation to the retroperitoneum. In mouse, rat, and dog, the duodenum was adhered to the mesocolon but not fixed to the retroperitoneum, thus theoretically there should be only one fold (the PDC) and no other folds or fossae. In pig, the anal side of the duodenum is too strongly and thickly fixed to the retroperitoneum to identify other folds or fossae. The morphologic pattern of the duodenojejunal flexure of pig could be classified as intermediate between human, rodent and dog. As with the IDF and the superior and inferior duodenal fossae, the suspensory muscle of the duodenum (ligament of Treitz) was found only in human, but was not macroscopically identified in the other animals. Since the suspensory muscle of the duodenum pulls the duodenojejunal flexure caudally to modulate the output through the duodenojejunal flexure, the muscle might be present only in bipeds, not in quadrupeds.

\section{CONCLUSIONS}

In summary, the SDF in human or PDC in animals and the adhesion between the duodenojejunal flexure and the mesocolon are preserved in all the examined animals and are relevant to the intestinal rotation. The fixation of the duodenum to the retroperitoneum may cause the differences in the anatomical structures, such as the IDF, the superior and inferior duodenal 
fossae, and the suspensory muscle of the duodenum, around the duodenojejunal flexure.

The present study is, to our knowledge, the first report about the comparative anatomy of the duodenojejunal flexure. Anatomical knowledge of the area is useful for duodenal and pancreatic surgeries, and for animal studies about the duodenum.

\section{REFERENCES}

1. Akin JT, Gray SW, Skandalakis JE. Vascular compression of the duodenum: Presentation of ten cases and review of the literature. Surgery. 1976; 79(5): 515-522, indexed in Pubmed: 1265660.

2. Barberini F, Zani A, Ripani M, et al. The complex arrangement of an "aorto-jejunal paraduodenal" fossa, as revealed by dissection of human posterior parietal peritoneum. Ann Anat. 2007; 189(3): 299-303, doi: 10.1016/j. aanat.2006.12.005, indexed in Pubmed: 17534040.

3. Crymble PT. The muscle of Treitz and the plica duodnojejunalis. Br Med J. 1910; 2: 1156-1159.

4. Desjardins AU. Left paraduodenal hernia. Ann Surg. 1918; 67(2): 195-201, indexed in Pubmed: 17863859.

5. Federative Committee on Anatomical Terminology (FCAT). Terminologia Anatomica: International Anatomical Terminology Thieme, Stuttgart. 1998: Germany.

6. Kent GC. Comparative anatomy of the Vertebrates. The C.V. Mosby Company, St Louis. 1983.

7. Konig $\mathrm{H}$, Liebich $\mathrm{H}$. Veterinary anatomy of domestic mammals: textbook and colour atlas (Japanese edition). Midori shobo. 2016: Tokyo.
8. Martin LC, Merkle EM, Thompson WM. Review of internal hernias: radiographic and clinical findings. AJR Am J Roentgenol. 2006; 186(3): 703-717, doi: 10.2214/AJR.05.0644, indexed in Pubmed: 16498098.

9. Meyers MA. Treitz redux: the ligament of Treitz revisited. Abdom Imaging. 1995; 20(5): 421-424, indexed in Pubmed: 7580775.

10. Netter FH. Atlas of human anatomy. (Japanese edition), 5th ed. Nankodo, Tokyo. 2011: Japan.

11. Nickel R, Schummer A, Seiferle E, et al. The viscera of the domestic mammals. Springer-Verlag, New York. 1979, doi: 10.1007/978-1-4757-6814-5.

12. Peltier J, Gars DLe, Page C, et al. The duodenal fossae: anatomic study and clinical correlations. Surg Radiol Anat. 2005; 27(4): 303-307, doi: 10.1007/s00276-005-0332-9, indexed in Pubmed: 16244781.

13. Romer AS. The vertebrate body. W. B. Saunders Company. 1970: Philadelphia.

14. Soffers JHM, Hikspoors JP, Mekonen HK, et al. The growth pattern of the human intestine and its mesentery. BMC Dev Biol. 2015; 15: 31, doi: 10.1186/s12861-015-0081-x, indexed in Pubmed: 26297675.

15. Sugiyama M, Suzuki Y, Nakazato T, et al. Intestinal derotation procedure for facilitating pancreatoduodenectomy. Surgery. 2016; 159(5): 1325-1332, doi: 10.1016/j. surg.2015.11.014, indexed in Pubmed: 26767309.

16. Treitz W. Ueber einen neuen Muskel am Duodenum des Menschen, über elastische Sehnnen, und einige andere anatomische Verhãltnisse. Vierteljahrsschrift fuer die praktishe Heilkunde. 1853; 37: 113-44.

17. World Association of Veterinary Anatomists.Nomina anatomica veterinaria. International Committee on Veterinary Anatomical Nomenclature. 2012. 